\title{
Exponential Dichotomies and Homoclinic Orbits from Heteroclinic Cycles*
}

\author{
Tiejun Chen, Yu Xiang, Weiwei Cai, Yuxiao Chen \\ Yiyang Medical College Hunan Pro of China, Yiyang, China \\ Email: cwwlove@sina.com
}

Received February 13, 2012; revised April 20, 2012; accepted April 28, 2012

\begin{abstract}
In this paper, we investigate the homoclinic bifurcations from a heteroclinic cycle by using exponential dichotomies. We give a Melnikov-type condition assuring the existence of homoclinic orbits form heteroclinic cycle. We improve some important results.
\end{abstract}

Keywords: Exponential Dichotomies; Homoclinic Orbits; Heteroclinic Cycle; Melnikov Function

\section{Introduction}

We consider the n-dimensional differential equations

$$
\dot{x}=f(x, v, \varepsilon)
$$

where $x \in R^{n}, \varepsilon$ is a small parameter, $v \in R^{2}$ is a parameter. In studying the global bifurcation, we usuaally assume unperturbed differential equations

$$
\dot{x}=f(x, 0,0)
$$

admits ahyperbolic equilibruim and a homoclinic orbit connecting it. It is the peresistence of homoclinic oribit and heteroclinic that we usually study in global bifurcation, we refer to Wiggins [1], Palmer [2,3], Naudot [4] and Meyer and Sell [5]. But in studying the pulses solutions of some recation-diffusion equations, we often meet the problem of homoclinic bifurcations from the heteroclinic cycles, refer to Kokubu [6], Chow, Deng and Terman [7], Gambaudo [8] and reference therein. Suppose Equation (1.2) has two hyperbolic equilibriums $p_{1}, p_{2}$ and two homoclinic orbits $q_{1}, q_{2}$ and two homoclinic orbits $q_{1}(t), q_{2}(t)$.

If

$$
\lim _{t \rightarrow-\infty} q_{i}(t)=p_{i}, \lim _{t \rightarrow+\infty} q_{i}(t)=p_{i+1}, \mathrm{I}=1,2
$$

(where we assume $q_{1}(t)-q_{3}(t) \quad p_{1}=p_{3}$ ) then we say that $\Gamma=q_{1}(t) \cup q_{2}(t) \cup q_{1} \cup q_{2}$ is a heteroclinic cycle consisting of $q_{1}(t), q_{2}(t), p_{1}$ and $p_{2}$. The study of homoclinic bifurcation from a heteroclinic cycle is very important and interest not only from the point of view of

"This work is supported by Program for Yiyang Municipal Bureau of Science and Technology of Hunan Pro in China (Ministry YKZ-2011 -30) bifurcation theory itself but also from the point of view of application, we refer to Kokubu [6], Chow, Deng and Terman [7]. The main purpose of this paper is to invertigate the homoclinic bifurcation from heteroclinic cycles by making use of exponential dichotomies and Melnikov technique. For convenience, we only discuss the case of heteroclinic cycles with length $=2$. Using the theory of exponential dichotomies, Melnikov functions and Slinikov chang of variable, Kokubu [6] investigate the periodic and homoclinic bifurcations from a heteroclinic cycle. In Kokubu [6], he needs to divide the problem into critical and non-critical two cases. Moreover, he needs that the heteroclinic orbits approach the hyperbolic equilibriums along the eignspaces associated with the principal eigenvalues. Chow, Deng and Terman [7] also studied the same problem in the non-critical case by making use of Liapunov-schmidt method and Silnikov's changes of variable and Poincare map and obtain some analytical results. Chow, Deng and Terman [7] also the conditions as in Kokubu [6]. Melnikov functions were not obtained in Chow, Deng and Terman [7]. Chow, Deng and Terman [9] studied the same problem as this paper, Kokubu [7] did not need to divide the problem into critical and non-critical two cases and unified the two cases and didn't ndde that the heteroclinic orbits approach the hyperbolic equilibriums along the eigenspaces associated with the principal eigenvalues. The results of Chow, Deng and Terman [9] are weaker than those of Kokubu [6] and Chow, Deng and Terman [7] under weaker assumptions because of the topological approachs.The purpose of this paper is to improve the above results by a analystic method (Lin's method [10]).We can also unify the critical and non-critical cases and weak the condi- 
tions of Kokubu [6], Chow, Deng and Terman [7,9]. Moreover, it is also an interesting to provide an analystic method of studying bifurcations of heteroclinic cycles. Many ideas of this paper come from Lin [10], Meyer and Sell [5], Kokubu [6] and Palmer [2,3]. But it should note that the results of this paper cannot be followed directly from these papers, much technique has been made. Let us finally mention the related results on the bifurcations of heteroclinic cycles. Sandstede [11] investigated the forced symmetry breaking of heteroclinic cycles. Guckenheimer and Holmes [12] discussed the spontaneous symmetry breaking of heteroclinic cycle. Krupa and Melbourne [13] studiecd the stability of heteroclinic cycle. On the other related results on heteroclinic cycles, we refer to the references of the above mentioned papers and good survey of Krupa [14]. The paper is organized as following. In Section 2, we give the main result; in Section 3, the proof of the main result is given.

The main tool used in this paper is theory of exponential dichotomies. We consider the linear differential equations

$$
\dot{x}=A(t)^{x}
$$

where $x \in R^{n}, \mathrm{~A}(\mathrm{t})$ is a $n \times n$ continuous bounded matrix on $R$. We say Equation (1.3) admits an exponential dichotomy on interval $\mathrm{J}$ if ther exist con stants $K, \alpha$, a projection $P$ and the fundamental matrix $\mathrm{X}(\mathrm{t})$ of Equation (1.3) satisfying;

$$
\begin{aligned}
& \left|X(t) P X^{-1}(s)\right| \leq K \mathrm{e}^{-a(t-s)} t \geq s \\
& \left|X(t)(I-P) x^{-1}(s)\right| \leq K \mathrm{e}^{-a(t-s)} \geq s
\end{aligned}
$$

for $t, s \in J$. On the theory of exponential dichotomies, refer to Coppel [15], Sacker and Sell [16] and Meyer and Sell [17]. On the relations between exponential dichotomies and homoclinic, heteroclinic bifurcations, we refer to Palmer [18] and Meyer and Sell [16].

\section{Main Result}

We consider differential equations

$$
\dot{x}=f(x, v, \varepsilon)
$$

where $x \in R^{n}, \varepsilon$ is small parameter, $v \in R^{2}$ is a parameter. $f: \Omega \times I_{1}^{2} \times I_{2} \rightarrow R^{n} i s C_{b}^{2}$ with respect to $(x, v, \varepsilon) \in \Omega \times I_{1}^{2} \times I_{2}$, where $\Omega \subset R^{2} a$ cllompact subset, $I_{1}$ a small interval containing zero, $I_{2}=[0, b]$ a small interval.

We assume C1. For $v=0, \varepsilon=0$, unperturbed equation

$$
\dot{x}=f(x, 0,0)
$$

Admits two hyperbolic equilibriums $p_{1}, p_{2}$ and two heteroclinic orbits $q_{1}(t), q_{2}(t)$ connecting $p_{1}, p_{2}$ respectively (form a heteroclinic cycle), that is,

$$
\begin{gathered}
\lim _{t \rightarrow-\infty} q_{2}(t)=p_{1}, \lim _{t \rightarrow \infty} q_{1}(t)=p_{2}, \\
\lim _{t \rightarrow-\infty} q_{2}(t)=p_{2}, \lim _{t \rightarrow+\infty} q_{2}(t)=p_{1}, . \\
q_{i}(t) \in \Omega, i=1,2 .
\end{gathered}
$$

We denote the heteroclinic cycle by

$$
\Gamma=q_{1}(t) \cup q_{2}(t) \cup q_{1} \cup q_{2} .
$$

We want to study under what conditions can a homoclinic orbit bifurcate from the heteroclinic cycle $\Gamma$ as the second case of Kokubu [6]

C2. All real parts of the matrix $f_{x}\left(p_{i}, 0,0\right)(i=1,2)$ are different from zero; and the number of eigenvalues with positive real parts is $m_{i}=m(<n)(i=1,2)$.

If the conditions $\mathrm{C} 1$ and $\mathrm{C} 2$ are satisfied then equation

$$
\dot{x}=f_{x}\left(p_{i}, 0,0\right) x, i=1,2 .
$$

admit an exponential dichotomy on both $R_{+}$and $R_{-}$, and the sum of dimensions of stable and unstable subspaces is $\mathrm{n}$. If follows from the roughness of exponential dichotomy that (refer to Zeng [12], Sacker and Sell [16], Coppel [15]) that the variational equations along $q_{i}(t)$

$$
\dot{x}=f_{x}\left(q_{i}(t), 0,0\right), i=1,2
$$

admit an exponential dichotomy on both $R_{+}$and $R_{-}$, and the sum of dimensions of the stable and unstable subspaces is $m_{1}+n-m_{2}=n$. In the follows, because we want to the exponent of $e^{\alpha \ln \varepsilon}=\varepsilon^{\alpha}$ to be greater that 1 , without loss of generality, we may assume the constants $K, \alpha>1$. Otherwise, we replace $l_{n} \varepsilon$ by $\frac{\alpha}{2} l_{n} \varepsilon$, then the exponent of $e^{\alpha \frac{2}{\alpha} \ln \varepsilon}=\varepsilon^{2}$ is greater than 1 .

C3. The variational Equations (2.4) admit a unique (up to a scalar multiple) nontrival bounded solution $\phi_{i}(t)$ on $R$.

Under the conditions C1, C2, C3, we can prove (refer to Zeng [12] that the adjoint equations of equations of (2.3), (2.4)

$$
\dot{\xi}=-g_{x}^{*}=\left(q_{i}(t)\right) \xi \mathrm{i}=1,2
$$

also admit unique (up to a scalar multiple ) nontrival bounded solution $\psi_{1}(t), \psi_{2}(t)$, respectively, on $\mathrm{R}$, and an exponential dichotomy on both $R_{+}$and $R_{-}$, respectively. The constants of the exponential dichotomies are also $K, \alpha$.

We let

$$
\begin{aligned}
& M_{1}=\int_{-\infty}^{+\infty} \psi_{2}^{*}(t) f_{v}\left(q_{1}(t), 0,0\right) \mathrm{d} t, \\
& M_{2}=\int_{-\infty}^{+\infty} \psi_{2}^{*}(t) f_{v}\left(q_{2}(t), 0,0\right) \mathrm{d} t .
\end{aligned}
$$

The main result of this paper is 
Theorem 1 We assume the conditions C2, C2 and C3 are satisfied, then when $\varepsilon, v$ sufficiently small Equation (2.1) admits a unique hyperbolic equilibrium $p_{2}(\varepsilon, v)$ satisfying $p_{1}(0,0)=p_{1}$.If the $2 \times 2$ matrix

$$
M=\left(\begin{array}{ll}
M_{1} & \\
& M_{2}
\end{array}\right)
$$

is invertible, the for $\varepsilon>0$ sufficiently small there exista a continuous function $v=v(\varepsilon)$ satisfying

$$
\mu(0)=\mu_{0}=-M^{-1}\left(\begin{array}{l}
\int_{-\infty}^{+\infty} \psi_{2}^{*}(t) f_{v}\left(q_{1}(t), 0,0\right) \mathrm{d} s \\
\int_{-\infty}^{+\infty} \psi_{2}^{*}(t) f_{v}\left(q_{2}(t), 0,0\right) \mathrm{d} s
\end{array}\right)
$$

such that the equation

$$
\dot{x}=f(t, \varepsilon v(\varepsilon), \varepsilon)
$$

admits a homoclinic orbit connecting $p_{1}(\varepsilon, \varepsilon v(\varepsilon))$ in the neighbourhood of the heteroclinic cycle $\Gamma$.

Remark If the conditions C1, C2 and C3 are satisfied, uing the standard method (refer to Zeng [19]), we can obtain the bifurcative equations of persistence of the two heteroclinic orbits $q_{1}(t)$ and $q_{2}(t)$

$$
\begin{aligned}
& \tilde{H}_{1}(\mu, \varepsilon)=\int_{-\infty}^{+\infty} \psi_{1}^{*}(s) g_{1}\left(s, \tilde{z}_{1}(s, \mu, \varepsilon), \mu, \varepsilon\right) \mathrm{d} s=0 \\
& \tilde{H}_{2}(\mu, \varepsilon)=\int_{-\infty}^{+\infty} \psi_{2}^{*}(s) g_{2}\left(s, \tilde{z}_{2}(s, \mu, \varepsilon), \mu, \varepsilon\right) \mathrm{d} s=0
\end{aligned}
$$

where $\tilde{z}_{i}(s, 0,0)=0, i=1,2$. If the matrix $\mathrm{M}$ is invertible then we can easily prove (refer to Zeng [19]) that for $\varepsilon \neq 0$ sufficiently small there exits a continuously differentiable function $\mu=\tilde{\mu}(\varepsilon)$ such that

$$
\tilde{H}(\varepsilon \tilde{\mu}(\varepsilon), \varepsilon)=0 \quad \tilde{H}_{2}(\varepsilon \tilde{\mu}(\varepsilon), \varepsilon)=0
$$

and

$$
\dot{x}=f(x, \varepsilon \tilde{\mu}(\varepsilon), \varepsilon)
$$

has two hyperbolic equilibriums $\tilde{p}_{1}(\varepsilon), \tilde{p}_{2}(\varepsilon)$, satisfying $\tilde{p}_{1}(0)=p_{1}$ and $\tilde{p}_{2}(0)=p_{2}$, and two heteroclinic orbits $\tilde{q}_{1}(t, \varepsilon), \quad \tilde{p}_{2}(t, \varepsilon)$ satisfying

$$
\begin{aligned}
& \lim _{t \rightarrow-\infty} \tilde{q}_{1}(t, \varepsilon)=\tilde{p}_{1}(\varepsilon) \lim _{t \rightarrow-\infty} \tilde{q}_{1}(t, \varepsilon)=\tilde{p}_{2}(\varepsilon), \\
& \lim _{t \rightarrow-\infty} \tilde{q}_{2}(t, \varepsilon)=\tilde{p}_{2}(\varepsilon) \lim _{t \rightarrow-\infty} \tilde{q}_{2}(t, \varepsilon)=\tilde{p}_{1}(\varepsilon) .
\end{aligned}
$$

That is, the heteroclinic cycle $\Gamma$ persists in the region of parameters

$$
\{(\varepsilon \tilde{\mu}(\varepsilon), \varepsilon)\}
$$

Fiom Theorem 1 of this paper we see that in the region of parameters

$$
\{(\varepsilon \mu(\varepsilon), \varepsilon)\}
$$

a homoclinic orbit connecting $p_{1}(\varepsilon, \varepsilon v(\varepsilon))$ bifurcates from the heteroclinic cycle $\Gamma$.

Kokubu [5] proved that

$$
\partial\{(\varepsilon \mu(\varepsilon), \varepsilon)\}=\{(\varepsilon \tilde{\mu}(\varepsilon), \varepsilon)\}
$$

We can also prove that if the conditions C1, C2 and C3 are satisfied then for $\varepsilon \neq 0$ sufficiently small a homoclinic orbit connecting $p_{2}(\varepsilon), P_{2}(0)=p_{2}$, bifurcates from the heteroclinic cycle $\Gamma$, but the region of parameters of bifurcation is different from $\{(\varepsilon \mu(\varepsilon), \varepsilon)\}$.

\section{The Proof of the Main Result}

To prove the main result of this paper, we want to find the bounded solutions of Equation (2.1) $x_{1}(t)$ on $(-\infty, \omega)$ and $x_{2}(t)$ on $(-\omega, \infty)$ satisfying

$$
x_{1}(\omega)=x_{2}(-\omega)
$$

We make a change of variables for Equation (2.1)

$$
\begin{aligned}
& x=z_{1}+q_{1}(t),-\infty \leq t<\omega . \\
& x=z_{2}+q_{2}(t),-\omega<t \leq \infty .
\end{aligned}
$$

respectively, and obtain the equations

$$
\begin{aligned}
& z_{1}=f\left(z_{1}+q_{1}(t), v, \varepsilon\right)-f\left(q_{1}(t), 0,0\right),-\infty<t \leq \omega . \\
& z_{2}=f\left(z_{2}+q_{2}(t), v, \varepsilon\right)-f\left(q_{2}(t), 0,0\right),-\omega \leq t<\infty .
\end{aligned}
$$

We write the above equations in the following form

$$
\begin{aligned}
& z_{1}=A_{1}(t) z_{1}+g_{1}\left(t, z_{1}, v, \varepsilon\right),-\infty<t \leq \omega . \\
& z_{2}=A_{2}(t) z_{2}+g_{2}\left(t, z_{2}, v, \varepsilon\right),-\omega \leq t<\infty .
\end{aligned}
$$

And the boundary value condition in the following form

$$
z_{1}(\omega)-z_{2}(-\omega)=q_{2}(-\omega)-q_{1}(\omega)
$$

where $\omega$ is sufficiently large.

$$
\begin{aligned}
& A_{i}(t)=f_{x}\left(q_{i}(t), 0,0\right) \\
& \begin{aligned}
g_{i}(t, z, v, \varepsilon) & =f\left(z_{1}+q_{i}(t), v, \varepsilon\right) \\
& -f\left(q_{5}(t), 0,0\right)-A_{i}(t) z_{i}
\end{aligned}
\end{aligned}
$$

$i=1,2 . \quad g_{i}\left(t, z_{i}, v, \varepsilon\right)=i=1,2$ satisfying:

$$
\left|g_{i}\left(t, z_{i}, v, \varepsilon\right)\right| \leq C_{1}\left(\left|z_{i}\right|^{2}+|v|+|\varepsilon|\right) i=1,2
$$

In order to find the bounded solutions of Equations (3.1), (3.2) and (3.3), we consider the following boundary value problem

$$
\begin{aligned}
& z_{1}=A_{1}(t) z_{1}+g_{1}\left(t, z_{1}, v, \varepsilon\right),-\infty<t \leq \ln \varepsilon . \\
& z_{2}=A_{2}(t) z_{2}+g_{2}\left(t, z_{2}, v, \varepsilon\right), \ln \varepsilon \leq t<\infty . \\
& z_{1}(-\ln \varepsilon)-z_{2}(\ln \varepsilon)=q_{2}(\ln \varepsilon)-q_{1}(-\ln \varepsilon) .
\end{aligned}
$$

where $\varepsilon<1$. For any $h_{1}(t), h_{2}(t) \in C_{b}^{2}\left(R, R^{n}\right)$, we first consider the following boundary value problems for $\varepsilon>0$ 


$$
\begin{gathered}
z_{1}=A_{1}(t) z_{1}+h_{1}(t),-\infty<t \leq-\ln \varepsilon . \\
z_{2}=A_{2}(t) z_{2}+h_{2}(t), \ln \varepsilon \leq t<\infty \\
z_{1}(-\ln \varepsilon)-z_{2}(\ln \varepsilon)=q_{2}(\ln \varepsilon)-q_{1}(-\ln \varepsilon)
\end{gathered}
$$

We let $b(\varepsilon)=q_{2}(\ln \varepsilon)-q_{1}(-\ln \varepsilon)$ and have the following lemma:

Lemma 1 Assume the conditions C1, C2 and C3 are satisfied.

Then there exists sufficiently small $\varepsilon_{0}>0$ such that for $0<\varepsilon \leq \varepsilon_{0}$ Equations (3.9), (3.10) and (3.11) admit a unque continuous except at $t=0$ bounded solution $z_{i}(t, \varepsilon)$ satisfying $\phi_{i}^{*}(0) z_{i}(0-, \varepsilon)=0, i=1,2$ with

$$
\left|z_{i}(t, \varepsilon)\right| \leq C_{2}\left(|b(\varepsilon)|+\left\|h_{i}(t)\right\|\right), i=1,2 .
$$

Moreover, $z_{i}(t, \varepsilon)$ is differentiable in $\varepsilon$ and with

$$
\left|z_{1 \varepsilon}(t, \varepsilon)\right|+\left|z_{2 \varepsilon}(t, \varepsilon)\right| \leq L \text {. }
$$

where $z_{i}(0-)$ denotes the left limit of function $z_{i}(t, \varepsilon)$ at $t=0, C_{1}$ is a constant independent of $\varepsilon$., Moreover, if

$$
\begin{aligned}
& \int_{-\infty}^{-\ln \varepsilon} \psi_{1}^{*}(s) h_{1}(s) \mathrm{d} s-\psi_{1}^{*}(-\ln \varepsilon) z_{1}(-\ln \varepsilon)=0 . \\
& \int_{\ln \varepsilon}^{-\ln \varepsilon} \psi_{2}^{*}(s) h_{2}(s) \mathrm{d} s-\psi_{2}^{*}(-\ln \varepsilon) z_{2}(-\ln \varepsilon)=0 .
\end{aligned}
$$

then $z_{1}(t), z_{2}(t)$ are continuous at $t=0$.

Proof Lemma 2 is mainly due to Lin [10]. For the proof of the first part of existences of the solutions $z_{i}(t, \varepsilon)$ satisfying (3.12), (3.13) and (3.14), we refer to Lin [10] and omit the proof. We now want to prove the second part that $z_{i}(t, \varepsilon)$ is differentiable in $\varepsilon$.We let $z_{1}(t, \varepsilon), z_{2}(t, \varepsilon)$ be the bounded solutions, which are continous except at $t=0$ and satisfy (3.12), (3.13) and (3.14), of equations

$$
\begin{aligned}
& z_{1}=A_{1}(t) z_{1}+h_{1}(t),-\infty<t \leq-\ln \varepsilon \\
& z_{2}=A_{2}(t) z_{2}+h_{2}(t), \ln \varepsilon \leq . t<\infty \\
& z_{1}(-\ln \varepsilon)-z_{2}(\ln \varepsilon)=q_{2}(\ln \varepsilon)-q_{1}(-\ln \varepsilon)
\end{aligned}
$$

Let $Z_{1}(t, \varepsilon), Z_{2}(t, \varepsilon)$ be the bounded solutions of equations

$$
\begin{aligned}
& Z_{1}=A_{1}(t) Z_{1},-\infty<t \leq-\ln \varepsilon . \\
& Z_{2}=A_{2}(t) Z_{2}, \ln \varepsilon \leq t<\infty .
\end{aligned}
$$

$$
Z_{1}(\ln \varepsilon)-Z_{2}(\ln \varepsilon)=\left\{A_{1}(-\ln \varepsilon) z_{1}(-\ln \varepsilon, \varepsilon)+A_{2}(\ln \varepsilon) z_{2}(\ln \varepsilon, \varepsilon)+A_{1}(-\ln \varepsilon) q_{1}(-\ln \varepsilon)+A_{2}(\ln \varepsilon) q_{2}(\ln \varepsilon)\right\} / \varepsilon
$$

Let

$$
\begin{aligned}
& \rho_{1}(t)=z_{1}(t, \varepsilon+h)-z_{1}(t, \varepsilon)-Z_{1}(t, \varepsilon) h, \\
& \rho_{2}(t)=z_{2}(t, \varepsilon+h)-z_{2}(t, \varepsilon)-Z_{2}(t, \varepsilon) h .
\end{aligned}
$$

then $\rho_{1}(t), \rho_{2}(t)$ are the solutions of equations

$$
\begin{aligned}
& \rho_{1}=A_{1}(t) \rho_{1},-\infty<t \leq-\ln \varepsilon . \\
& \rho_{2}=A_{2}(t) \rho_{2}, \ln \varepsilon \leq t<\infty . \\
& \rho_{1}(-\ln \varepsilon)-\rho_{2}(\ln \varepsilon)=z_{1}(-\ln \varepsilon, \varepsilon+h) \\
& -z_{2}(\ln \varepsilon, \varepsilon+h)-z_{1}(-\ln \varepsilon, \varepsilon) \\
& +z_{2}(\ln \varepsilon, \varepsilon)-Z_{1}(-\ln \varepsilon, \varepsilon) h+Z_{2}(\ln \varepsilon, \varepsilon) h .
\end{aligned}
$$

In the same method as follows, we can show that

$$
\begin{aligned}
& \quad\left|\eta_{1}(t)\right|+\left|\eta_{2}(t)\right|=O(|h|) \\
& \qquad\left|\eta_{1}(t, \varepsilon)\right|+\left|\eta_{2}(t, \varepsilon)\right| \leq 2 C \\
& \text { its a constant } L>0 \text { such that } \\
& \left|z_{1 \varepsilon}(t, \varepsilon)\right|+\left|z_{2 \varepsilon}(t, \varepsilon)\right| \leq L .
\end{aligned}
$$$$
\left|\eta_{1}(t, \varepsilon)\right|+\left|\eta_{2}(t, \varepsilon)\right| \leq 2 C_{1}\left|z_{1}(-\ln \varepsilon, \varepsilon+h)-z_{2}(\ln \varepsilon, \varepsilon+h)-z_{1}(-\ln \varepsilon, \varepsilon)+z_{2}(\ln \varepsilon, \varepsilon)\right|
$$

hence there exsits a constant $L>0$ such that

This completes the proof of Lemma 2.

Now we consider Equations (3.1)-(3.3). We have the following lemama:

Lemma2 Assume conditions C1, C2 and C3 are satisfied. Then there exist sufficiently small $\varepsilon_{0}>0$ and the

$$
z_{i \varepsilon}(t, \varepsilon)=Z_{i}(t, \varepsilon), i=1,2 .
$$

Now we prove the boundness of $z_{i \varepsilon}$. Let

$$
\begin{aligned}
& \eta_{1}(t, \varepsilon)=z_{1}(t, \varepsilon+h)-z_{1}(t, \varepsilon), \\
& \eta_{2}(t, \varepsilon)=z_{2}(t, \varepsilon+h)-z_{2}(t, \varepsilon),
\end{aligned}
$$

then $\eta_{1}(t, \varepsilon), \eta_{2}(t, \varepsilon)$ are the solutions of equations

$$
\begin{gathered}
\eta_{1}=A_{1}(t) \eta_{1},-\infty<t \leq-\ln \varepsilon . \\
\quad \eta_{1}=A_{1}(t) \eta_{1},-\infty<t \leq-\ln \varepsilon . \\
\eta_{1}(-\ln \varepsilon, \varepsilon)-\eta_{2}(\ln \varepsilon, \varepsilon) \\
=z_{1}(-\ln \varepsilon, \varepsilon+h)-z_{n}(\ln \varepsilon, \varepsilon+h) \\
-z_{1}(-\ln \varepsilon, \varepsilon)+z_{2}(\ln \varepsilon, \varepsilon)
\end{gathered}
$$

From (3.12) we obtain constants $C_{2}, L>0$ such that for $0<\varepsilon \leq \varepsilon_{0}$ Equations (3.1)-(3.3) admit aunque continuous except at $t=0$ bounded solution $z_{i}(t, v, \varepsilon)$ satisfying

$$
\phi_{i}^{*}(0) z_{i}(0-, v, \varepsilon)=0, i=1,2
$$

with

$$
\begin{aligned}
\left|z_{i}(t, v, \varepsilon)\right| & \leq C_{2}(|b(\varepsilon)|+|v|+|\varepsilon|) \\
& \leq C_{2}\left(\left|2 K \varepsilon^{\alpha}\right|+|v|+|\varepsilon|\right), i=1,2
\end{aligned}
$$




$$
\left|z_{i v}(t, v, \varepsilon)\right|+\left|z_{i \varepsilon}(t, v, \varepsilon)\right| \leq L, i=1,2 .
$$

Moreover, if

$$
\begin{aligned}
G_{1}(v, \varepsilon)= & \int_{-\infty}^{-\ln \varepsilon} \psi_{1}^{*}(s) g_{1}\left(s, z_{1}(s, v, \varepsilon), v, \varepsilon\right) \mathrm{d} s \\
& -\psi_{1}^{*}(-\ln \varepsilon) z_{1}(-\ln \varepsilon, v, \varepsilon)=0 \\
G_{1}(v, \varepsilon)= & \int_{-\infty}^{-\ln \varepsilon} \psi_{1}^{*}(s) g_{2}\left(s, z_{2}(s, v, \varepsilon), v, \varepsilon\right) \mathrm{d} s \\
& -\psi_{1}^{*}(-\ln \varepsilon) z_{2}(-\ln \varepsilon, v, \varepsilon)=0
\end{aligned}
$$

then $z_{1}(t, v, \varepsilon), z_{2}(t, v, \varepsilon)$ are continuous at $t=0$.

The proof of Lemma 2 can be proved by contract fixed point theorem and is similar to that of Lin [10].

From Lemma 2 we see that if we have proved that bifurcative Equations (3.16) and (3.17) can be can be solved then we find the continuously bounded solutions of Equations (3.1), (3.2) and (3.3)

$$
z_{1}(t, v, \varepsilon)-\infty<t \leq-\ln \varepsilon
$$

and

$$
z_{2}(t, v, \varepsilon), \ln \varepsilon \leq t<\infty .
$$

Now we mainly solve bifurcative Equations (3.16) and (3.17). We make a change of variable for Equations (3.16) and (3.17) $v \sim \varepsilon v$ and obtain the following bifurcative equation

$$
\begin{aligned}
B_{1}(v, \varepsilon) & =G_{1}(v, \varepsilon) \\
& =\int_{-\infty}^{-\ln \varepsilon} \psi_{1}^{*}(s) g_{1}\left(s, z_{1}(s, v, \varepsilon), v, \varepsilon\right) \mathrm{d} s \\
& -\psi_{1}^{*}(-\ln \varepsilon) z_{1}(-\ln \varepsilon, v, \varepsilon)=0 \\
B_{1}(v, \varepsilon)= & G_{1}(v, \varepsilon) \\
= & \int_{-\infty}^{-\ln \varepsilon} \psi_{1}^{*}(s) g_{2}\left(s, z_{2}(s, v, \varepsilon), v, \varepsilon\right) \mathrm{d} s \\
& -\psi_{1}^{*}(-\ln \varepsilon) z_{2}(-\ln \varepsilon, v, \varepsilon)=0
\end{aligned}
$$

From (3.15) we have

$$
\left|z_{i}(t, v, \varepsilon)\right| \leq C_{2}\left(2 K|\varepsilon|^{\alpha}+|\varepsilon||v|+|\varepsilon|\right) i=1,2
$$

Leting $\varepsilon \rightarrow 0$ in the above equation, we obtain

$$
z_{i}(t, 0,0)=0 \quad i=1,2
$$

(Remark ACTUALLY, $z_{i}(t, \varepsilon v, \varepsilon)$ is defined only for $\varepsilon>0$. but due to the existence of its limit, here we define the vaule of the limit to be the value at $\varepsilon=0$. In the sequel, we make the same definition.)

From the property of $\psi_{i}(t)$ we have

$$
\left|\psi_{i}(\ln \varepsilon)\right| \leq K e^{\alpha \ln \varepsilon}=K \varepsilon, i=1,2
$$

hence

$$
\lim _{t \rightarrow-\infty} \psi_{i}(-\ln \varepsilon)=0, i=1,2
$$

From the representation of (3.18), (3.19), (3.21) and
(3.33) we obtain

$$
\begin{aligned}
B_{1}(v, 0)= & \int_{-\infty}^{\infty} \psi_{1}^{*}(s) g_{1}\left(s, z_{1}(s, 0,0), 0,0\right) \mathrm{d} s \\
& -\lim _{\varepsilon \rightarrow 0} \psi_{1}^{*}(-\ln \varepsilon) z_{1}(-\ln \varepsilon, \varepsilon v, \varepsilon) \\
= & \int_{-\infty}^{\infty} \psi_{1}^{*}(s) g_{1}\left(s, z_{1}(s, 0,0,0) \mathrm{d} s\right)=0
\end{aligned}
$$

In the same way, we can obtain

$$
B_{2}(v, 0)=\int_{-\infty}^{\infty} \psi_{2}^{*}(s) g_{2}\left(s, z_{1}(s, 0,0,0) \mathrm{d} s\right)=0
$$

For convenience, we define a $2 \times 1$ matrix

$$
B(v, \varepsilon)=\left(\begin{array}{l}
B_{1}(v, \varepsilon) \\
B_{2}(v, \varepsilon)
\end{array}\right)
$$

then we have

$$
B(v, 0)=0
$$

We define

$$
H(\varepsilon, v)=\left(\begin{array}{l}
\frac{B(v, \varepsilon)}{\varepsilon}, \varepsilon \neq 0 \\
B_{\varepsilon}(v, \varepsilon), \varepsilon=0
\end{array}\right)
$$

Obviously, for $\varepsilon \neq 0$ equation

$$
B(\varepsilon, v)=0
$$

And equation

$$
H(\varepsilon, v)=0
$$

equivalent. Now we want to find the solutions of Equation (3.26). We first compute $B_{\varepsilon}(v, 0)$. From (3.18) we have

$$
\begin{aligned}
& B(\varepsilon, v) \\
= & -\psi_{2}(-\ln \varepsilon) \frac{1}{\varepsilon} g_{1}\left(-\ln \varepsilon, z_{1}(-\ln \varepsilon, \varepsilon v, \varepsilon), \varepsilon v, \varepsilon\right) \\
& +\int_{-\infty}^{\ln \varepsilon} \psi_{1}^{*}(s) \frac{\mathrm{d}}{\mathrm{d} \varepsilon} g_{1}\left(s, z_{1}(s, \varepsilon v, \varepsilon), \varepsilon v, \varepsilon\right) \mathrm{d} s \\
& -\frac{\mathrm{d}}{\mathrm{d} \varepsilon}\left\{\psi_{1}^{*}(-\ln \varepsilon) z_{1}(-\ln \varepsilon, \varepsilon v, \varepsilon)\right\}
\end{aligned}
$$

Now we compute (3.27). Since

$$
\left|g_{i}(t, z, \varepsilon v, \varepsilon)\right| \leq C_{1}\left(\left|z_{1}\right|^{2}+|\varepsilon||v|+|\varepsilon|\right)
$$

we have

$$
\begin{aligned}
& \left|\frac{1}{\varepsilon} g_{1}\left(-\ln \varepsilon, z_{1}(-\ln \varepsilon, \varepsilon v, \varepsilon), \varepsilon v, \varepsilon\right)\right| \\
& \leq \frac{1}{\varepsilon} C_{1}\left(\left|z_{1}-\ln \varepsilon, \varepsilon v, \varepsilon\right|^{2}+|\varepsilon||v|+|\varepsilon|\right) \\
& \leq \frac{1}{\varepsilon} C_{2}\left[C_{2}\left(2 K e^{\alpha-\frac{1}{2}}+|\varepsilon||v|+|\varepsilon|\right)^{2}|\varepsilon||v|+|\varepsilon|\right] \\
& \leq C_{2}\left[C_{1}\left(2 K e^{\alpha-\frac{1}{2}}+|\varepsilon|^{\frac{1}{2}}|v|+|\varepsilon|^{\frac{1}{2}}\right)^{2}|v|+1\right]
\end{aligned}
$$


and hence $\frac{1}{\varepsilon} g_{1}\left(-\ln \varepsilon, z_{1}(-\ln \varepsilon, \varepsilon v, \varepsilon), \varepsilon v, \varepsilon\right)$ is bounded for $\varepsilon \geq 0$.

Since

$$
\lim _{\varepsilon \rightarrow 0} \psi_{1}^{*}(-\ln \varepsilon)+\psi_{1}^{*}(\infty)=0,
$$

we have

$$
\begin{aligned}
& \lim _{\varepsilon \rightarrow 0} \psi_{1}^{*}(-\ln \varepsilon) \frac{1}{\varepsilon} g_{1}(-\ln \varepsilon), z_{1}(-\ln \varepsilon, \varepsilon v, \varepsilon), \varepsilon v, \\
& \varepsilon=0
\end{aligned}
$$

Noting $z_{1}(t, 0,0)=0$, we can easily prove that

$$
\begin{aligned}
& \lim _{\varepsilon \rightarrow 0} \frac{\mathrm{d}}{\mathrm{d} \varepsilon} g_{1}\left(s, z_{1}(-\ln \varepsilon, \varepsilon v, \varepsilon), \varepsilon v, \varepsilon\right) \\
& =f_{v}\left(q_{1}(s), 0,0\right) v+f_{\varepsilon}\left(q_{1}(s), 0,0\right),
\end{aligned}
$$

hence

$$
\begin{aligned}
& \left.\lim _{\varepsilon \rightarrow 0} \int_{-\infty}^{-\ln \varepsilon} \psi_{1}^{*}(s) \frac{\mathrm{d}}{\mathrm{d} \varepsilon} g_{1}\left(s, z_{1}(-\ln \varepsilon, \varepsilon v, \varepsilon)\right) \varepsilon v, \varepsilon\right) \mathrm{d} s \\
& =\int_{-\infty}^{-\ln \varepsilon} \psi_{1}^{*}(s) f_{v}\left(q_{1}(s), 0,0\right) v+f_{\varepsilon}\left(q_{1}(s), 0,0\right) \mathrm{d} s .
\end{aligned}
$$

Last, since

$$
\begin{aligned}
& \frac{\mathrm{d}}{\mathrm{d} \varepsilon}\left\{\psi_{1}^{*}(-\ln \varepsilon) z_{1}(-\ln \varepsilon, \varepsilon v, \varepsilon)\right\} \\
& \leq\left|\psi_{1}^{*}(-\ln \varepsilon) f_{x}\left(q_{1}(-\ln \varepsilon)\right) \frac{2}{\varepsilon} z_{1}(-\ln \varepsilon, \varepsilon v, \varepsilon)\right| \\
& +\mid \psi_{1}^{*}(-\ln \varepsilon)\left[f\left(z_{1}(-\ln \varepsilon, \varepsilon v, \varepsilon)+q_{1}(-\ln \varepsilon) \varepsilon v, \varepsilon\right)\right. \\
& \left.-f\left(q_{1}(-\ln \varepsilon), 0,0\right)\right] \frac{2}{\varepsilon} \\
& +\left|\varepsilon z_{1 v}(-\ln \varepsilon, \varepsilon v, \varepsilon)\right|+\left|z_{1 \varepsilon}(-\ln \varepsilon, \varepsilon v, \varepsilon)\right| \\
& \leq K \| z_{1}(t, \varepsilon v, \varepsilon)|| \varepsilon^{\alpha-1}+\left|\psi_{1}(-\ln \varepsilon)\right| \\
& {\left[C_{1}\left(\left|z_{1}(-\ln \varepsilon, \varepsilon v, \varepsilon)\right|+|\varepsilon||v|+|\varepsilon|\right)\right] \frac{2}{\varepsilon}+L} \\
& \leq K \| z_{1}(t, \varepsilon v \varepsilon)|| \varepsilon^{\alpha-1} \\
& +\left|\psi_{1}(-\ln \varepsilon)\right|\left[C_{1}\left(C_{1}|v|+1\right)+1+|v|\right]+L
\end{aligned}
$$

we obtain

$$
\lim _{\varepsilon \rightarrow 0} \frac{\mathrm{d}}{\mathrm{d} \varepsilon}\left\{\psi_{1}^{*}(-\ln \varepsilon) z_{1}(-\ln \varepsilon, \varepsilon v, \varepsilon)\right\}=0
$$

From (3.28), (3.29) and (3.31) we have

$$
\begin{aligned}
B_{1 \varepsilon}(v, 0) & =\int_{-\infty}^{+\infty} \psi_{1}^{*}(s)\left(f_{v}\left(q_{1}(s), 0,0\right) v\right. \\
& +f_{\varepsilon}\left(q_{1}(s), 0,0\right) \mathrm{d} s
\end{aligned}
$$

In the same way, we can prove

$$
\begin{aligned}
B_{2 \varepsilon}(v, 0) & =\int_{-\infty}^{+\infty} \psi_{1}^{*}(s)\left(f_{v}\left(q_{2}(s), 0,0\right) v\right. \\
& +f_{\varepsilon}\left(q_{2}(s), 0,0\right) \mathrm{d} s
\end{aligned}
$$

Hence we have

$$
\begin{aligned}
& H(v, 0)=B_{\varepsilon}(v, 0)=\left(\begin{array}{l}
B_{1 \varepsilon}(v, 0) \\
B_{2 \varepsilon}(v, 0)
\end{array}\right) \\
& =\left(\begin{array}{l}
\int_{-\infty}^{+\infty} \psi_{1}^{*}(s)\left(f_{v}\left(q_{1}(s), 0,0\right) v+f_{\varepsilon}\left(q_{1}(s), 0,0\right) \mathrm{d} s\right) \\
\int_{-\infty}^{+\infty} \psi_{1}^{*}(s)\left(f_{v}\left(q_{2}(s), 0,0\right) v+f_{\varepsilon}\left(q_{2}(s), 0,0\right) \mathrm{d} s\right)
\end{array}\right) \\
& =\left(\begin{array}{l}
M_{1} \\
M_{2}
\end{array}\right) v+\left(\begin{array}{l}
\int_{-\infty}^{+\infty} \psi_{1}^{*}(s)\left(f_{v}\left(q_{1}(s), 0,0\right) \mathrm{d} s\right) \\
\int_{-\infty}^{+\infty} \psi_{2}^{*}(s)\left(f_{v}\left(q_{2}(s), 0,0\right) \mathrm{d} s\right)
\end{array}\right)
\end{aligned}
$$

Let

$$
v_{0}=-M^{-}\left(\begin{array}{l}
\int_{-\infty}^{+\infty} \psi_{1}^{*}(s)\left(f_{\varepsilon}\left(q_{1}(s), 0,0\right) \mathrm{d} s\right) \\
\int_{-\infty}^{+\infty} \psi_{2}^{*}(s)\left(f_{\varepsilon}\left(q_{2}(s), 0,0\right) \mathrm{d} s\right)
\end{array}\right)
$$

then we have

$$
H_{v}\left(v_{0}, 0\right)=B_{\varepsilon}\left(v_{0}, 0\right)=0
$$

From (3.34) we have

$$
H_{v}\left(v_{0}, 0\right)=M
$$

Since the matrix $\mathrm{M}$ is invertible, it follows from the implicit function theorem that for $\varepsilon>0$ sufficienly small there exists a continuous function $v=v(\varepsilon)$, $v(0)=v_{0}$ satisfying

$$
H(v(\varepsilon), \varepsilon)=0
$$

Hence for $\varepsilon>0$ sufficiently small we have

$$
B(v(\varepsilon), \varepsilon)=0
$$

Hence for $\varepsilon>0$ sufficiently small Equations (3.6), (3.7) and (3.8)

$$
\begin{aligned}
& z_{1}(t, \varepsilon)=z_{1}(t, \varepsilon v(\varepsilon), \varepsilon),-\infty<t \leq-\ln \varepsilon, \\
& z_{1}(t, \varepsilon)=z_{1}(t, \varepsilon v(\varepsilon), \varepsilon), \ln \varepsilon<t \leq \infty,
\end{aligned}
$$

So for $\varepsilon>0$ sufficiently small the equation

$$
\dot{x}=f(t, \varepsilon v,(\varepsilon), \varepsilon) \text {. }
$$

has two solutions

$$
\begin{aligned}
& x_{1}=(t, \varepsilon)=z_{1}(t, \varepsilon)+q_{1}(t),-\infty<t \leq-\ln \varepsilon, \\
& x_{2}=(t, \varepsilon)=z_{2}(t, \varepsilon)+q_{2}(t), \ln \varepsilon<t \leq \infty,
\end{aligned}
$$

satisfying

$$
x_{1}(-\ln \varepsilon, \varepsilon)=x_{2}(-\ln \varepsilon, \varepsilon)
$$

We construct a solution of Equation (3.38) by making use of $x_{1}(t, \varepsilon)$ and $x_{2}(t, \varepsilon)$

$$
x(t, \varepsilon)=\left(\begin{array}{l}
x_{1}(t-\ln \varepsilon, \varepsilon),-\infty<t \leq 0 \\
x_{2}(t-\ln \varepsilon, \varepsilon), 0<t \leq \infty
\end{array}\right.
$$


Since $x_{1}(-\ln \varepsilon, \varepsilon)=x_{2}(-\ln \varepsilon, \varepsilon), \quad x(t, \varepsilon)$ is a continuously bounded solution of Equation (3.38).

Now we show $x(t, \varepsilon)$ is a homoclinic orbit connecting the equilibrium $q_{1}(\varepsilon, \varepsilon v(\varepsilon))$. Since when $-\infty<t \leq 0$

$\left|x_{1}(t, \varepsilon)-q_{1}(\varepsilon, \varepsilon v(\varepsilon))\right|$

$=\left|z_{1}(t-\ln \varepsilon, \varepsilon v(\varepsilon), \varepsilon)+q_{1}(t-\ln \varepsilon)-q_{1}(\varepsilon, \varepsilon v(\varepsilon))\right|$

$\leq\left|z_{1}(t-\ln \varepsilon, \varepsilon v(\varepsilon), \varepsilon)\right|+\left|q_{1}(t-\ln \varepsilon)-q_{1}\right|$

$+\left|q_{1}(\varepsilon, \varepsilon v(\varepsilon))-q_{1}\right|$

Hence for any $\delta>0$, there exist $\varepsilon_{0}>0$ and $T>0$ such that when $0<\varepsilon \leq \varepsilon_{0}$ and $t<-T+\ln \varepsilon$, we have

$$
\left|x_{1}(t, \varepsilon)-q_{1}(\varepsilon, \varepsilon v(\varepsilon))\right| \leq \delta
$$

Since $q_{1}(\varepsilon, \varepsilon v(\varepsilon))$ is hyperbolic, we obtain (refer to [9]) for $\varepsilon \neq 0$ sufficiently small

$$
\lim _{t \rightarrow-\infty} x(t, \varepsilon)=q_{1}(\varepsilon, \varepsilon v(\varepsilon))
$$

In the same way, we can prove that

$$
\lim _{t \rightarrow \infty} x(t, \varepsilon)=q_{1}(\varepsilon, \varepsilon v(\varepsilon))
$$

Hence $x(t, \varepsilon)$ is a homoclinic orbit connecting $q_{1}(\varepsilon, \varepsilon v(\varepsilon))$ in the neighbouthood of the heteroclinic cycle $\Gamma$.

Theorem 1 discussed the second case of bifurcations of Kokubu [6]. Acutally, we slso investigate the first case of bifurcation as in Figure 2 in the same way and have the following result. We assume

B1 for $v=0, \varepsilon=0$, unperturbed equation

$$
\dot{x}=f(x, 0,0)
$$

Admits three hyperbolic equilibriums $p_{1}, p_{2}, p_{3}$ and two heteroclinic orbits $q_{1}(t), q_{2}(t)$ connecting $p_{1}$ to $p_{2}, p_{2}$ to $p_{3}$, respectively, that

$$
\begin{aligned}
& \lim _{t \rightarrow-\infty} q_{1}(t)=p_{1}, \lim _{t \rightarrow \infty} q_{1}(t)=p_{2} \\
& \lim _{t \rightarrow-\infty} q_{2}(t)=p_{2}, \lim _{t \rightarrow \infty} q_{2}(t)=p_{3}
\end{aligned}
$$

We denote by $\Pi=q_{1}(t) \cup q_{2}(t) \cup q_{1} \cup q_{2} \cup p_{3}$.

Theorem 2 We assume the conditions B1, C2 and C3 are satisfied, then when $\varepsilon, \rho$ sufficiently sall Equation (1.1) admits two hyperbolic equilibrium $p_{1}(\varepsilon, \rho)$, $p_{2}(\varepsilon, \rho)$ satisfying $p_{1}(0,0)=p_{1}, \quad p_{2}(0,0)=p_{2}$. If the $2 \times 2$ matrix

$$
M=\left(\begin{array}{l}
M_{1} \\
M_{2}
\end{array}\right)
$$

Is invertible, then for $\varepsilon>0$ sufficiently small there exists a continuous function $\rho=\rho(\varepsilon)$ satisfying

$$
\rho(0)=-M^{-1}\left(\begin{array}{l}
\int_{-\infty}^{+\infty} \psi_{1}^{*}(s)\left(f_{\varepsilon}\left(q_{1}(s), 0,0\right) \mathrm{d} s\right) \\
\int_{-\infty}^{+\infty} \psi_{2}^{*}(s)\left(f_{\varepsilon}\left(q_{2}(s), 0,0\right) \mathrm{d} s\right)
\end{array}\right)
$$

Such that the equation

$$
\dot{x}=f(t, \varepsilon \rho(\varepsilon), \varepsilon)
$$

Admits a heteroclinic orbit connecting $p_{1}(\varepsilon, \varepsilon \rho(\varepsilon))$ to $p_{3}(\varepsilon, \varepsilon \rho(\varepsilon))$ in the neighbourhood of the heteroclinic cycle $\Pi$.

\section{REFERENCES}

[1] S. Wiggins, "Golbal Bifurcations and Chaos," SpringerVerlag, New York, 1988

[2] K. J. Palmer, "Transversal Heteroclinic Orbits and Cherry’s Example of a Nonintegrable Hamiltonian System,” Journal of Differential Equations, Vol. 65, No. 3, 1986, pp. 321-360. doi:10.1016/0022-0396(86)90023-9

[3] K. J. Palmer, "Exponential Dichotomies and Transversal Homoclinic Points,” Journal of Differential Equations, Vol. 55, No. 2, 1984, pp. 225-256. doi:10.1016/0022-0396(84)90082-2

[4] S. Campbell and P. Holmes, "Bifurcation from O(2)sy Mmetric Heterclinic Cycles with Three Interacing Mofes," Nonlinearity, Vol. 4, 1991, pp. 697-726.

[5] K. R. Meyer and G. R. Sell, "Melnikov Transforms, Bernoulli Bundle and Almost Periodic Perturbations," Transactions of the American Mathematical Society, Vol. 314, No. 1, 1989, pp. 63-105.

[6] H. Kokubu, "Homoclinic and Heteroclinic Bifurcations of Vector Fields,” Japan Journal of Industrial and Applied Mathematics, Vol. 5, No. 3, 1988, pp. 455-501. doi:10.1007/BF03167912

[7] S. N. Chow, B. Deng and D. Terman, "The Bifurcations of a Homoclinic and a Periodic Orbit from Two Heteroclinic Orbits," SIAM Journal on Mathematical Analysis, Vol. 21, No. 1, 2000, pp. 179-204. doi:10.1137/0521010

[8] J. M. Gambaudo, P. Glendinning and C. Tresser, "Collages de Cycles et Suites de Farey," Comptes Rendus de l'Académie des Sciences, Vol. 299, 1984, pp. 711-714.

[9] S. N. Chow, B. Deng and D. Terman, "The Bifurcation of a Homoclinic Orbit from Two Heteroclinic Orbits-A Topological Approach," Applicable Analysis: An International Journal, Vol. 42, No. 1-4, 1991, pp. 1057-1080. doi:10.1080/00036819108840047

[10] X. B. Lin, "Using Melnikov’s Method to Solve Silnikov Problems," Proceedings of the Royal Society of Edinburgh: Section A Mathematics, Vol. 116, No. 3-4, 1990, pp. 295-325. doi:10.1017/S0308210500031528

[11] B. Sandstede and A. Scheel, "Forced Symmetry Breaking of Homoclinic Cycles,” Nonlinearity, Vol. 8, No. 3, 2009, pp. 333-365. doi:10.1088/0951-7715/8/3/003

[12] J. Guckenheimer and P. Holmes, "Strucarrlly Stable Pulse Heteroclinic Cycles," Mathematical Proceedings of the Cambridge Philosophical Society, Vol. 103, No. 1, 2008, 
pp. 189-192. doi:10.1017/S0305004100064732

[13] M. Krupa and I. Melbourne, "Asymptotic Stability of Heteroclinic Cycles in Systems with Symmetry," Proceedings of the Royal Society of Edinburgh: Section A Mathematics, Vol. 134, No. 6, 2004, pp. 1177-1197. doi:10.1017/S0308210500003693

[14] M. Krupa, "Robust Heteroclinic Cycles,” Journal of Nonlinear Science, Vol. 7, No. 2, 2011, pp. 129-176. doi:10.1007/BF02677976

[15] W. A. Coppel, "Dichotomies in Stability Theory, Lecture Notes in Mathematics,” Springer-Verlag, New York, 1978.

[16] R. J. Sacker and G. R. Sell, “A Spectral Theory for Linear Differential Systems," Journal of Differential Equations, Vol. 27, No. 3, 1978, pp. 320-385. doi:10.1016/0022-0396(78)90057-8

[17] P. Chossat, M. Krupa, I. Melbourne and A. Scheel, “Transverse Bifurcations of Homkoclinic Cycles,” Physica D: Nonlinear Phenomena, Vol. 100, No. 1-2, 2011, pp. 85100. doi:10.1016/S0167-2789(96)00186-8

[18] V. Naudot, "Hyperbolic Dynamice in the Unfolding of a Degenerate Homoclinic Orbit,” Preprint.

[19] W. Y. Zeng, "Exponential Dichotomies and Transversal Homoclinic Orbits in Degenerate Cases,” Journal of Dynamics and Differential Equations, Vol. 7, No. 4, 1995, pp. 521-548. doi:10.1007/BF02218723

[20] G. R. Sell, "Bifurcation of Higher Dimensional Tori," Archive for Rational Mechanics and Analysi, Vol. 69, No. 3, 1979, pp. 199-230. doi:10.1007/BF00248134 